\title{
SUDDEN CHANGES IN THE INTENSITY OF HIGH ENERGY X-RAYS FROM SCO X-1
}

\author{
P. C. AGRAWAL, S. BISWAS, G. S. GOKHALE, V. S. IYENGAR, P. K. KUNTE, \\ R. K. MANCHANDA, and B. V.SREEKANTAN \\ Tata Institute of Fundamental Research, Bombay 5, India
}

In this note we wish to report briefly the observation of sudden changes in the intensity of Sco X-1 by a factor of about 3 recorded in the energy interval $29.9-52.3 \mathrm{keV}$ on December 22, 1968 between $04 \mathrm{~h} 27 \mathrm{~m}$ and $05 \mathrm{~h} 53 \mathrm{~m}$ UT. The observation was made with an X-ray telescope flown in a balloon from Hyderabad, India. The balloon was launched at $0200 \mathrm{hr}$ UT and reached the ceiling of $7.5 \mathrm{~g} / \mathrm{cm}^{2}$ of residual atmosphere at $0435 \mathrm{hr}$ UT. The X-ray telescope consisted of a NaI(T1) crystal with an area of $97.3 \mathrm{~cm}^{2}$ and thickness $4 \mathrm{~mm}$, surrounded by both active and passive collimators. The telescope was mounted on an oriented platform which was programmed to look in four specified directions successively, of azimuths, $\phi=0^{\circ}, 110^{\circ}, 180^{\circ}$ and $310^{\circ}$ $\left(\phi=0^{\circ}\right.$ being North and $\phi=90^{\circ}$, West), spending about $4 \mathrm{~min}$ in each direction during a cycle of period of about $16 \mathrm{~min}$. The axis of the telescope was inclined at an angle of $32^{\circ}$ with respect to the zenith. A pair of crossed flux gate magnetometers provided information every $8.2 \mathrm{sec}$ on the azimuth of the telescope. The pulse heights from the $X$-ray detector were sorted into several channels extending from 10 to $120 \mathrm{keV}$. An $\mathrm{Am}^{241}$ source came into the field of view of the telescope once in $15 \mathrm{~min}$ for about $30 \mathrm{sec}$ to provide in-flight calibration of the detector. The meridian transit of Sco X-1 was at $0454 \mathrm{hr}$ UT. Just before the balloon reached the ceiling Sco X-1 was in the field of view of the telescope for $3 \mathrm{~min}$ and $41 \mathrm{sec}$. After the balloon reached ceiling, Sco X-1 was in the field of view of the telescope on five occasions between 0443 and $0553 \mathrm{hr}$ UT. During the last observation, however, the balloon had lost altitude by about $1 \mathrm{~g} / \mathrm{cm}^{2}$. The excess counts due to Sco X-1 were obtained by subtracting the counting rates corresponding to the North direction which did not include any known $\mathrm{X}$-ray sources. The observation on Sco X-1 in the 1 st cycle was made while the balloon was still ascending and consequently the interposed grammage was changing from 10.5 to $9.7 \mathrm{~g} / \mathrm{cm}^{2}$. However, for the energy range under consideration, the change in the background counting rate was not significant and there cannot be any doubt regarding the genuineness of the excess counts recorded.

In Figure 1, we have plotted the observed intensity of Sco X-1 corrected for atmospheric attenuation in the energy interval $29.9-52.3 \mathrm{keV}$ as a function of time. The dashed line (1) represents the weighted mean intensity of all the first five observations and the dashed line (2) represents the weighted mean intensity of the 2nd, 3rd and 4th observations.

It is clear from the figure that the observed intensities deviate considerably from dashed line (1), suggesting that the mean is not a good fit for the data. (The probability level is 0.05 .) On the other hand dashed line (2) is a good fit for the 2 nd, 3rd and 4 th 


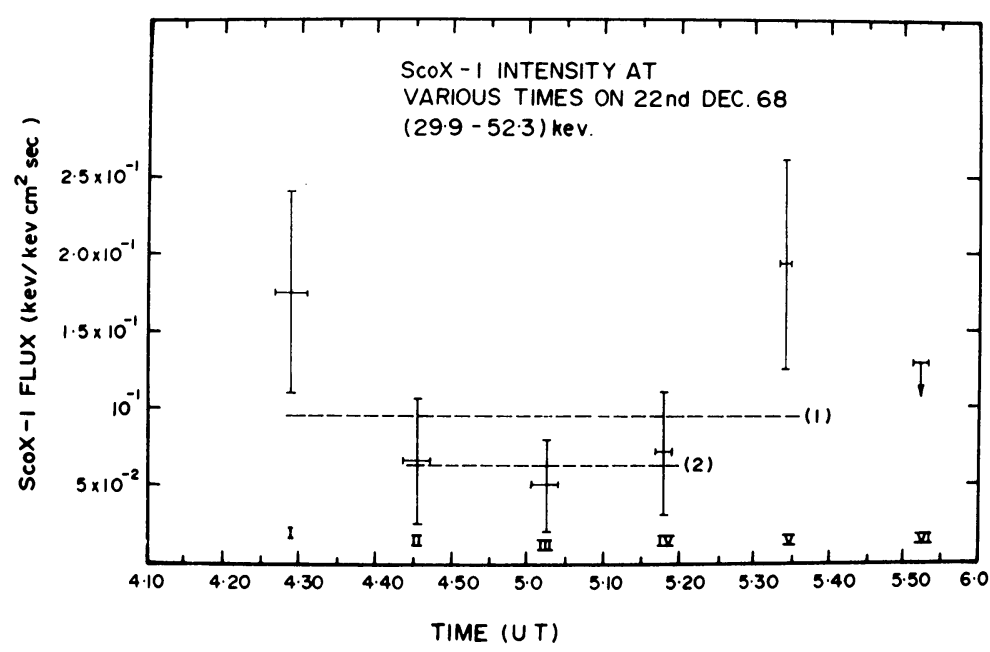

Fig. 1. Intensity variations of Sco X-1 at various times on December 27,1968 , in the energy interval of $29.9-52.3 \mathrm{keV}$.

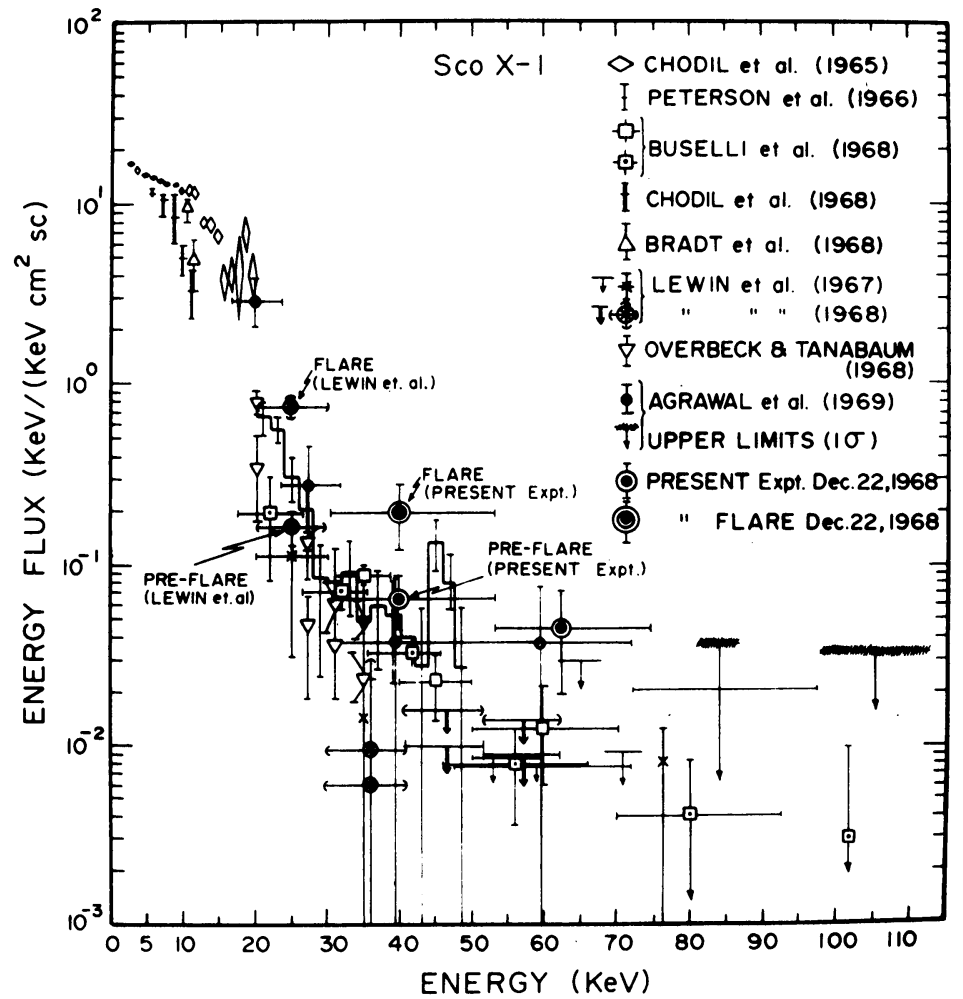

Fig. 2. The pre-flare and flare-time intensities of Sco X-1 on December 22, 1968, plotted with available spectral data of Sco X-1. 
observations. These two features make us believe that the intensity of Sco X-1 in the energy range 29.9-52.3 keV has not remained at the same level during the entire period of our observation extending over $90 \mathrm{~min}$. The intensity has first decreased by a factor of about 3, and remained constant for some time, and again shot up by a factor of 3 and decreased again - the rise and fall occurring in times less than about $15 \mathrm{~min}$. For this reason the 5 th observation may be called a flare.

The absolute flux values corresponding to the mean of 2 nd, 3rd and 4th observations for two energy channels 29.9-52.3 and 52.3-74.7 keV, have been plotted in Figure 2, where we have summarised the existing spectral data (1-9) on Sco X-1. In the same figure we have also plotted the flux value recorded in the energy channel 29.9-52.3 keV during the 5th cycle (designated - flare present). The flare observed in this experiment is in some respects similar to one reported by Lewin et al. (1968). A fourfold increase was observed by them in the energy channel $20-30 \mathrm{keV}$ at an atmospheric depth of $3.5 \mathrm{~g} / \mathrm{cm}^{2}$.

It is clear from Figure 2 that there is considerable spread in the flux values of Sco X-1 recorded by different observers at different times. A few of the observations at least have been attributed to long term (several months) variations in the intensity of Sco X-1 by Overbeck and Tananbaum (1968). While our pre-flare value corresponding to the energy channel $29.9-52.3 \mathrm{keV}$ falls within the range of previously reported values, the flux corresponding to the flare point is higher by almost a factor of 5. We cannot say whether the higher intensity recorded during the 1 st observation is again due to a flare or whether the intensity had remained high for a considerable period of time. However, the fact that the intensity dropped within $15 \mathrm{~min}$ by a factor of 3 to a value close to the generally reported values, favours the possibility of another flare having occurred during the 1st observation also.

Further details of these observations are reported in Nature 224, 51, 1969.

\section{References}

Agrawal, P. C., Biswas, S., Gokhale, G. S., Iyengar, V. S., Kunte, P. K., Manchanda, R. K., and Sreekantan, B. V.: 1969 (this proceedings).

Bradt, H., Naranan, S., Rappaport, S., and Spada, G.: 1968, Astrophys. J. Lett. 152, 1005.

Buselli, G., Clancy, M. C., Davison, P. J. N., Edwards, P. J., McCracken, K. G., and Thomas, R. M.: 1968, Nature 219, 1124.

Chodil, G., Jopson, R. C., Mark, H., Seward, F. D., and Swift, C. D.: 1965, Phys. Rev. Lett. 15, 605. Chodil, G., Mark, H., Rodrigues, R., Seward, F. D., Swift, C. D., Turiel, I., Hiltner, W. A., Wallerstein, G., and Mannery, E. J.: 1968, Astrophys. J. Lett. 154, 645.

Lewin, W. H. G., Clark, G. W., and Smith, W. B.: 1967, Astrophys. J. Lett. 150, L153.

Lewin, W. H. G., Clark, G. W., and Smith, W. B.: 1968, Astrophys. J. Lett. 152, L55.

Overbeck, J. W. and Tananbaum, H. D.: 1968, Astrophys. J. Lett. 153, 899.

Peterson, L. E. and Jacobson, A. S.: 1966, Astrophys. J. Lett. 145, 62. 